\title{
Les matériaux biomimétiques
}

\author{
Corinne Chevallard et Patrick Guenoun \\ LIONS (Laboratoire Interdisciplinaire sur l'Organisation Nanométrique et Supramoléculaire), \\ Service de Chimie Moléculaire, Bât. 125, \\ C.E.A. Saclay, 91191 Gif-sur-Yvette Cedex
}

\begin{abstract}
Les matériaux du vivant présentent souvent des propriétés mécaniques remarquables, inégalables en laboratoire en raison de leurs conditions très particulières d'élaboration. Nombre d'entre eux sont des matériaux composites, alliant phases organique et minérale, dont l'organisation structurale est hiérarchisée. La biominéralisation est un des processus constitutifs de ces matériaux où, comme dans la nacre, une phase cristalline croît en interaction avec une matrice organique. La tentative de reproduire ces conditions de minéralisation au laboratoire dans des systèmes modèles simples conduit à proposer différents scénarios et modèles de couplage que cet article passe en revue, en se focalisant essentiellement sur les matériaux analogues à la nacre.
\end{abstract}

Les matériaux biomimétiques sont nés de l'observation du vivant et, plus précisément, de l'observation de certains matériaux que la nature a dotés de propriétés inaccessibles aux matériaux artificiels conventionnels. Le biomimétisme, démarche par laquelle on cherche à " copier » le vivant, s'est imposé comme une voie prometteuse de synthèse des matériaux : la reproduction des mécanismes d'élaboration des matériaux vivants, ou tout au moins de leur structure, doit permettre l'obtention de matériaux aux propriétés voisines. Les matériaux biomimétiques peuvent en particulier servir de biomatériaux (os ou peau artificiels, par exemple) [1].

Ces objets posent au physicien de réels défis : sous des conditions « modérées " ou « douces » de température, de pression ou de $\mathrm{pH}$, l'auto-assemblage de briques élémentaires (polymères, tensioactifs, particules minérales) bien choisies permet-il de reproduire les organisations hiérarchiques observées dans la nature ? Avons-nous acquis, grâce aux progrès des vingt dernières années sur les systèmes moléculaires organisés, une maîtrise suffisante pour comprendre et reproduire des matériaux vivants ? Ce sont ces questions que nous souhaitons confronter ici à quelques résultats ou approches récents qui illustreront plus concrètement les problèmes physico-chimiques rencontrés dans ces études. Nous introduirons la problématique des matériaux biomimétiques grâce à l'exemple de la nacre. Au-delà de ses particularités, cet exemple illustre de manière assez générale les démarches suivies par les physiciens qui s'intéressent aux matériaux biomimétiques.

\section{Organisation structurale et conditions d'élaboration des matériaux du vivant}

L'intérêt suscité auprès de la communauté scientifique par les matériaux du vivant s'explique par plusieurs de leurs caractéristiques :

- tout d'abord, ils présentent un haut degré d'organisation, qui se traduit par l'existence de structures hiérarchiques emboîtées, comme c'est le cas par exemple pour l'os (cf. figure 1) ou la carapace de crabe [2]. La plus petite échelle d'organisation est souvent nanométrique. Comment décrypter les processus mis en œuvre et reproduire ces structures en laboratoire?

- en second lieu, la richesse de ces matériaux résulte de la possibilité qu'a la nature de concevoir des matériaux adaptés à des contraintes extérieures variées. Les araignées produisent ainsi différents types de fils, répondant à divers besoins, tels que le déplacement, la protection de leurs œufs, la construction de l'armature de leur toile ou la capture des proies. L'araignée $N$. clavipes possède notamment deux types de fils dont la différence de composition protéique se traduit par des valeurs de déformation à la rupture de 9 et $30 \%$ respectivement. Comment s'inspirer des matériaux biologiques pour appréhender les ingrédients minimaux conduisant à des propriétés voulues (relation structure - propriétés)?

- enfin, ces matériaux sont obtenus dans des conditions environnementales modérées (température, pression, valeur de $\mathrm{pH})$. À l'inverse, les techniques traditionnelles de fabrication humaine font souvent appel à des conditions extrêmes de température, de pression ou de $\mathrm{pH}$, conditions coûteuses à réaliser et qui ne permettent pas d'obtenir les mêmes matériaux.

L'organisation structurale fait intervenir des processus d'auto-assemblage capables d'organiser le matériau à des échelles aussi petites que le nanomètre par l'intermédiaire d'une grande densité d'interfaces aux interactions spécifiques (voir l'exemple de l'os sur la figure 1) [3]. On parle alors de construction bottom-up, où l'on imagine une mise en place successive de structures de taille croissante et de caractéristiques physico-chimiques variées. Cette référence fournit un premier schéma opératoire " simple ", en opposition à un mode top-down de structuration progressive d'un objet macroscopique (tel un sculpteur partant d'un bloc homogène sans structure pour en faire un objet présentant de nombreux détails mais composé d'un matériau unique). La structuration ascendante des matériaux biologiques est en fait compliquée par l'existence de couplages entre les différentes échelles,

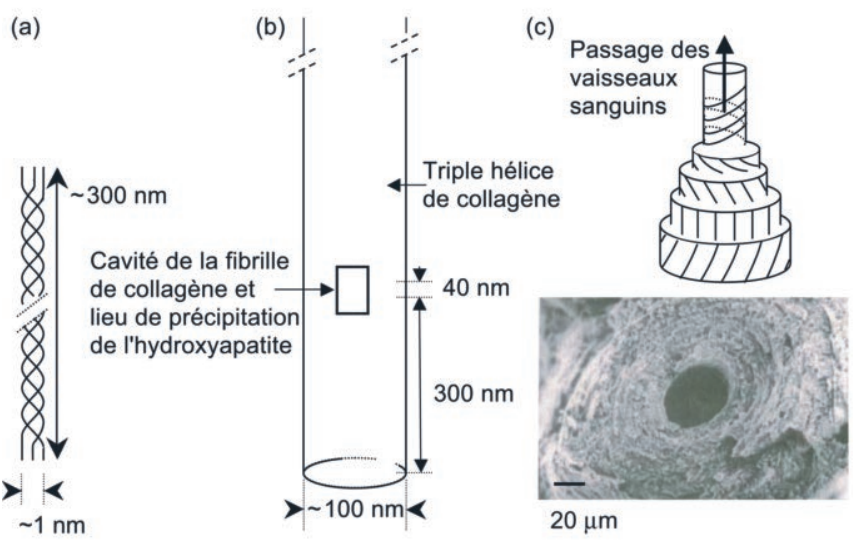

Figure 1. Structures hiérarchiques de l'os. a) Le filament de tropocollagène est constitué de trois polypeptides assemblés en triple hélice (échelle du nanomètre). b) Des fibrilles de collagène (échelle de la centaine de nanomètres) se développent par association des filaments précédents et précipitation d'hydroxyapatite dans les cavités de la structure. c) Dans la partie dense de l'os (os cortical), les fibrilles se structurent à leur tour en lamelles concentriques autour des canaux de passage des vaisseaux sanguins. Chaque lamelle est constituée de fibrilles de collagène se développant en hélice autour du canal, avec une orientation différente pour chaque lamelle. (Photographie de S. Weiner et H.D. Wagner, 1998, Annual Review of Materials Science 28, 271-298). 
couplages indispensables au maintien de la structure du matériau et, probablement, à ses propriétés spécifiques ${ }^{(1)}$.

Les connaissances acquises dans le domaine des processus d'auto-assemblage au cours des dernières décennies ${ }^{(2)}$ [4] laissent aujourd'hui espérer une compréhension et une reproduction des mécanismes d'élaboration des matériaux biologiques, grâce à la mise en œuvre de certaines stratégies de synthèse biomimétique, du type bottom-up. Cette recherche s'est développée depuis une vingtaine d'années sous une forme pluridisciplinaire associant biologistes, chimistes, physiciens et spécialistes des matériaux.

\section{Les biocomposites}

Parmi les matériaux biologiques, nombreux sont ceux qualifiés de "biocomposites ", associant une phase organique, formée notamment de protéines, à une phase inorganique minérale. L'os figure parmi ces biocomposites et associe des cristaux d'hydroxyapatite $\left(\mathrm{Ca}_{10}\left(\mathrm{PO}_{4}\right)_{6}(\mathrm{OH})_{2}\right)$ aux fibres de collagène dont l'organisation a été décrite dans la figure 1. La nacre, matière iridescente qui couvre la partie interne des coquilles de certains mollusques (voir figure de couverture), correspond quant à elle à un assemblage de protéines, polysaccharides et tablettes de carbonate de calcium $\left(\mathrm{CaCO}_{3}\right)$. L'association intime des composantes organique et inorganique confère à ces structures hybrides des caractéristiques mécaniques tout à fait exceptionnelles, analogues à celles des composites conventionnels, pouvant être modulées par une variation des proportions des deux composantes (stœchiométrie minéral-organique) ou par l'inclusion d'eau (cas de la nacre).

\section{La biominéralisation}

L'observation des matériaux biocomposites met en évidence l'aptitude des systèmes biologiques à réguler (donc à " contrôler ") la précipitation de certains minéraux et à organiser la phase minérale sous forme de structures hiérarchiques parfois très élaborées (cf. figure 2) [5].

Les minéraux rencontrés dans les matériaux biologiques (60 différents sont répertoriés) sont majoritairement le carbonate de calcium (coquilles de mollusques, exosquelettes de micro-organismes, capteurs de gravité), le phosphate de cal-

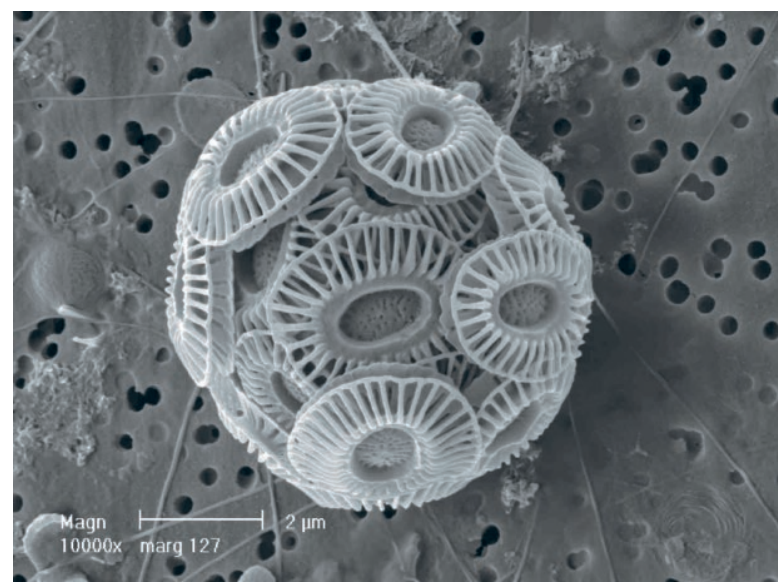

Figure 2. Coccosphère: squelette calcitique de l'algue unicellulaire Emiliana huxleyi (barre d'échelle $=2 \mu \mathrm{m}$ ). Crédit photographique : Jeremy Young.

(1) Par exemple, beaucoup d'efforts sont actuellement entrepris pour comprendre comment les contraintes mécaniques macroscopiques exercées sur l'os régulent l'activité des cellules osseuses (mécanotransduction)

(2) L'auto-assemblage moléculaire s'effectue sous l'influence de forces d'interactions (de type Van der Waals, électrostatique ou liaison hydrogène) d'énergie comparable à l'énergie thermique, $\mathrm{k}_{\mathrm{B}} \mathrm{T}$. Les molécules s'assemblent entre elles à 1,2 ou 3 dimensions en formant des structures supramoléculaires, dont les faibles énergies de liaison autorisent de nombreux réarrangements en fonction de contraintes extérieures. cium (os, dents - émail et ivoire), la silice amorphe (exosquelette des diatomées) et l'oxyde de fer [3].

Dans de nombreux cas, la structure minérale élaborée est différente de celle qui serait observée en l'absence de contrôle : la nacre est ainsi formée de carbonate de calcium sous une forme cristalline (aragonite) différente de la forme thermodynamiquement la plus stable dans les conditions standards (calcite). On observe aussi la précipitation de silice amorphe dans les dents de certains gastéropodes marins (berniques). Mais le contrôle exercé sur la minéralisation ne s'arrête pas à la sélection de la variété cristalline : l'ensemble des processus de nucléation et de croissance est régulé par l'organisme. La biominéralisation correspond ainsi à un mécanisme de précipitation dirigée, à partir d'une phase liquide, qui sélectionne le lieu de minéralisation ("régiosélectivité »), fixe l'orientation cristallographique de croissance, et définit la morphologie du cristal (organisation spatiale).

Le contrôle de la biominéralisation semble réalisé par le biais de molécules organiques biologiques (protéines, polysaccharides, etc.), certaines riches en groupements acides, sécrétées par des cellules spécifiques de l'organisme. L'autoorganisation d'une catégorie de molécules conduit à l'élaboration d'une matrice organique dont les interfaces avec l'environnement aqueux sont les lieux de la précipitation. Certaines des molécules impliquées induisent très probablement des variations de concentrations ioniques, créant localement des conditions de sursaturation par rapport au minéral. D'autres pourraient jouer le rôle d'inhibiteur et réguler ainsi la croissance du cristal ou sélectionner un type cristallin particulier en inhibant la croissance d'une autre forme cristalline. Les composants organiques majoritaires, insolubles, constituent l'armature organique support des molécules de contrôle. Cette armature peut être détruite au cours de la minéralisation, comme c'est le cas pour l'émail dentaire.

\section{Un exemple de biocomposite : la nacre}

La nacre illustre bien l'ensemble des phénomènes évoqués plus haut. II s'agit d'un matériau hybride formé de tablettes polygonales d'aragonite (une forme cristalline du carbonate de calcium $\mathrm{CaCO}_{3}$ ) insérées dans une matrice organique (fines couches de protéines-polysaccharides). Cette matrice organique se compose d'un échafaudage insoluble, fait principalement de protéines fibrillaires, et de protéines acides solubles adsorbées sur cet échafaudage. Les tablettes d'aragonite, d'une épaisseur d'environ $0,5 \mu \mathrm{m}$ et d'une extension latérale de 5 à $10 \mu \mathrm{m}$, s'organisent sous forme de plans séparés par des couches organiques de $30 \mathrm{~mm}$ d'épaisseur (voir figure 3 ou encore la photo de couverture pour un exemple de nacre colonnaire). Chaque tablette d'aragonite est polycristalline, et l'existence de grains de quelques dizaines de nanomètres de taille a pu être mise en évidence par microscopie à force atomique sur une nacre de gastéropode. Si l'orientation de croissance des tablettes correspond bien à la direction de croissance rapide de l'aragonite en volume, la forme finale du cristal ne ressemble en rien à l'aragonite synthétique : celle-ci se développe généralement sous forme d'aiguilles et non sous forme de tablettes. On voit ici un des nombreux aspects du contrôle évoqué plus haut.

La matrice polymérique joue un rôle important dans la résistance mécanique du matériau : elle arrête la propagation des fractures en dissipant l'énergie associée, par déformation de la phase organique. La nacre présente ainsi un travail de fracture, perpendiculairement au plan des couches, 3000 fois plus élevé que l'aragonite pure. Ces propriétés mécaniques remarquables seraient notamment dues à l'existence, chez certaines molécules organiques, de domaines de fort repliement. Les liaisons intra-moléculaires à l'origine de ce repliement seraient successivement rompues lors du travail de déformation du matériau, limitant ainsi la déformation du cristal minéral. 
(a)

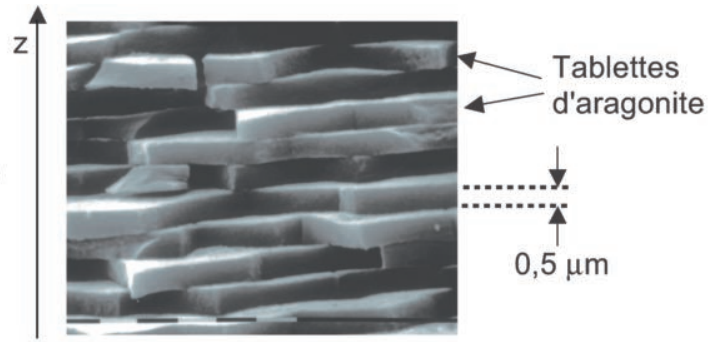

(b)

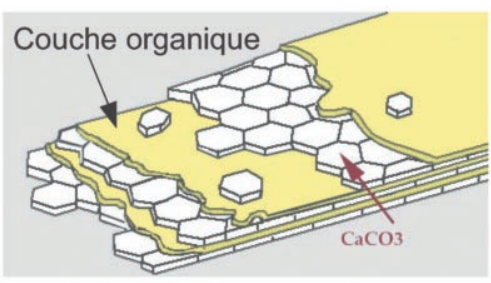

(c)

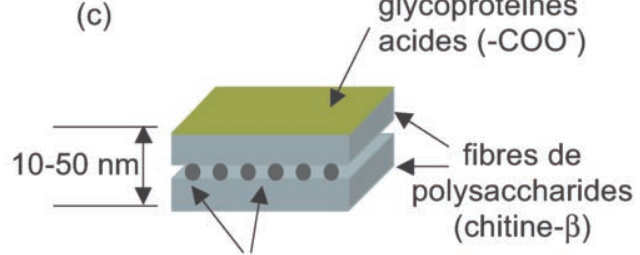

protéines fibrillaires

(silk-fibroin)

Figure 3. Structure de la nacre : a) photographie par microscopie électronique de la nacre en coupe verticale (permission de J.P. Cuif et Y. Dauphin, UMR 8148 IDES, Université de Paris 11) - Échelle : un trait blanc $=1 \mu \mathrm{m}$; b) représentation schématique de la structure alternée organique / inorganique de la nacre (crédit : cours de Berkeley) ; c) composition de la couche organique.

La nacre a fait l'objet de très nombreuses études dans les vingt dernières années, sans doute à cause de la relative simplicité de sa structure, ainsi qu'en raison de ses caractéristiques mécaniques exceptionnelles qui la placent devant les meilleures céramiques que nous sommes aujourd'hui capables de synthétiser. Par ailleurs, la nacre apparaît comme un matériau biocompatible ostéointégrable qui pourrait permettre la régénération et le comblement osseux, et éviter ainsi certaines étapes de chirurgie consistant à enlever des plaques intermédiaires qui promeuvent la régénération.

Afin d'évoquer avec un maximum de clarté les questions posées au physicien par l'observation des matériaux biologiques, nous consacrerons la suite de cet article à l'étude de la nacre. Nous décrirons en particulier les expériences réalisées pour mieux comprendre les mécanismes de son élaboration, ainsi que les tentatives de synthèse de matériaux qu'elle a inspirées.

\section{Modélisation de la croissance de la nacre et scénarios de minéralisation}

Si l'implication des couches organiques dans le contrôle de la minéralisation de la nacre ne fait aucun doute, la nature exacte de cette implication reste difficile à cerner. La présence de protéines présentant des groupements acides (groupements carboxyliques) à la surface des couches organiques a pu être démontrée. Ces groupements, qui portent une charge négative, pourraient constituer des sites privilégiés de nucléation du minéral. Faut-il dès lors réduire la biominéralisation au processus de précipitation au contact d'une surface organique chargée négativement ? C'est ce que supposent un grand nombre d'études expérimentales récentes. Afin de parvenir à une modélisation, ces études ignorent volontairement l'aspect tridimensionnel de l'élaboration de la nacre, ainsi que la possibilité d'une croissance couplée de la phase inorga-

nique minérale et de la matrice organique. En dépit - ou à cause - de ces simplifications déjà importantes, nous allons voir que le scénario de minéralisation et les conditions de sa mise en œuvre restent difficiles à définir.

Les études citées précédemment considèrent une couche organisée de molécules organiques, synthétiques ou biologiques, choisies pour leur groupement fonctionnel acide $\left(\mathrm{CO}_{2} \mathrm{H}, \mathrm{SO}_{3} \mathrm{H}\right.$, groupement aspartique des protéines, etc.) et pour leur nature amphiphile ${ }^{(3)}$. Le caractère amphiphile des molécules permet de les déposer sous forme d'une monocouche à l'interface entre une solution aqueuse et l'air (film de Langmuir, cf. figure 4), ou encore sur un substrat, mimant imparfaitement dans les deux cas l'attachement à une matrice insoluble. Les groupements hydrophiles ionisables des molécules organiques plongent dans une solution aqueuse (appelée « sous-phase ») sursaturée en carbonate de calcium. Les conditions de minéralisation de la nacre sont ainsi schématiquement reproduites. Les paramètres de contrôle de ces expériences sont alors les concentrations ioniques de la solution, l'organisation spatiale de la monocouche et l'état d'organisation des têtes polaires.

Les têtes polaires étant acides, la monocouche forme un plan chargé négativement (tête polaire ionisée, $\mathrm{CO}_{2}^{-}$par exemple) qui attire les ions $\mathrm{Ca}^{2+}$ par interactions électrostatiques, induisant ainsi une augmentation de la valeur de sursaturation au voisinage de la monocouche. Les ions $\mathrm{Ca}^{2+}$ favorisent aussi l'apparition d'une phase cristallisée du tensioactif. La nucléation du cristal intervient dès lors pour une valeur critique de la sursaturation. L'analyse des différentes expériences permet plusieurs constatations :

- en présence d'une monocouche, la précipitation du carbonate de calcium se produit préférentiellement au contact de la surface organique. II est donc possible d'imposer le lieu de minéralisation par la distribution spatiale de la phase organique ;

- l'analyse ex situ des cristaux, en général réalisée par microscopie électronique à balayage et par diffraction de rayons $\mathrm{X}$, révèle que les cristaux ont une orientation préférentielle : la face parallèle à la monocouche correspond à un plan cristallographique spécifique. Cette orientation varie avec la composition chimique et la structure spatiale du film organique. La surface organique permet donc de contrôler l'orientation cristallographique du minéral ;

- la variété cristalline apparue dépend elle aussi de la nature chimique des molécules organiques. Moins stables que la calcite, la vatérite et l'aragonite peuvent cependant être obtenues par le choix judicieux de molécules organiques formant une monocouche de structure adaptée [6, 7].

En dépit de sa simplicité, la configuration expérimentale retenue permet donc de reproduire certains éléments de la

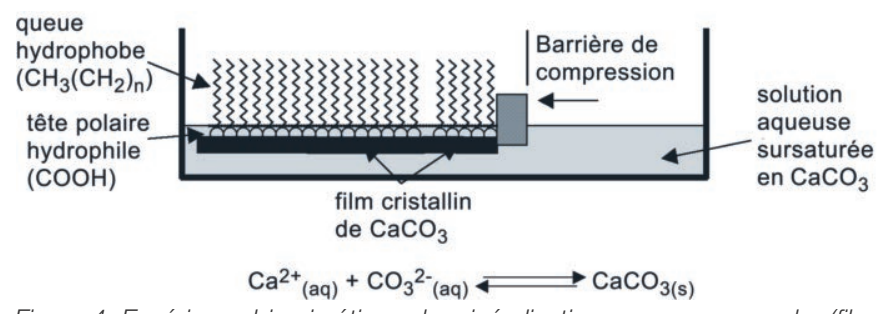

Figure 4. Expérience biomimétique de minéralisation : une monocouche (film de Langmuir) est formée à l'interface entre l'air et une solution aqueuse sursaturée en $\mathrm{CaCO}_{3}$. La précipitation se produit préférentiellement au voisinage de la monocouche.

(3) Une molécule amphiphile est formée de deux parties d'affinités différentes vis-à-vis des solvants. L'une d'elles présente une bonne affinité pour les solvants polaires tels que l'eau : il s'agit en général d'un groupement fonctionnel ionisable, $-\mathrm{CO}_{2} \mathrm{H}$ ou $-\mathrm{SO}_{3} \mathrm{H}$ par exemple, désigné par l'expression « tête polaire ». L'autre partie est soluble dans les milieux apolaires : on parle alors souvent de «queue hydrophobe " comme dans le cas d'une chaîne aliphatique $\mathrm{CH}_{3}\left(\mathrm{CH}_{2}\right)_{n}$ (cf. figure 4). 
biominéralisation : régiosélectivité, orientation cristallographique et sélection de la variété cristalline. Comment comprendre l'origine de ce contrôle et le comparer aux mécanismes mis en œuvre in vivo ? Un premier modèle suppose que le cristal germe à partir du film organique selon un mécanisme " hétérogène " (par opposition à une nucléation volumique " homogène " liée à des fluctuations thermiques). La nucléation hétérogène est favorisée si la disposition des molécules organiques dans le plan reproduit avec un faible écart la maille cristalline du minéral (relation d'épitaxie entre la phase minérale et la phase organique). Suivant cette hypothèse, la variété cristalline sélectionnée est celle qui présente la meilleure homologie de structure de l'un de ses plans calcium avec le plan organique (distances commensurables entre les plans denses du calcium et de la phase organique). La même condition fixerait l'orientation du germe cristallin [6].

Le choix de la variété cristalline et de son orientation spatiale pourrait également être dicté par un effet stéréochimique. $\mathrm{Si}$ la disposition spatiale des groupements anioniques de la monocouche reproduit la disposition des ions carbonates d'une phase minérale donnée, la nucléation de cette phase sera favorisée vis-à-vis de toutes les autres. Cet effet pourrait expliquer que sous une monocouche d'acide stéarique $\left(\mathrm{CH}_{3}\left(\mathrm{CH}_{2}\right)_{16} \mathrm{COOH}\right)$ on observe la croissance de vatérite, préférentiellement à la calcite favorisée par l'épitaxie [7] (figure 5).

Toutefois, seules des techniques d'analyse sophistiquées peuvent permettre de valider un scénario de nucléation hétérogène suivie d'une croissance du cristal. Des expériences de diffraction de rayons $X$ in situ, menées sur le système expérimental précédent d'acide stéarique [8], ont permis d'observer le phénomène de minéralisation aux temps courts : la précipitation intervient en fait à proximité de la monocouche, où la sursaturation est plus élevée, et produit des cristaux désorientés (poudre) qui se réorientent au cours du temps au contact du film organique par des effets de tension de surface. Si la nucléation hétérogène n'a pour l'instant jamais été observée avec le carbonate de calcium, elle a en revanche pu être mise en évidence pour le minéral $\mathrm{BaF}_{2}$ précipité au contact d'un film organique à l'interface liquide/air (épitaxie).

Certaines molécules biologiques interviennent aussi comme inhibiteurs ou catalyseurs de la réaction de précipitation. L'ajout d'acide polyacrylique en sous-phase dans l'expérience précé-
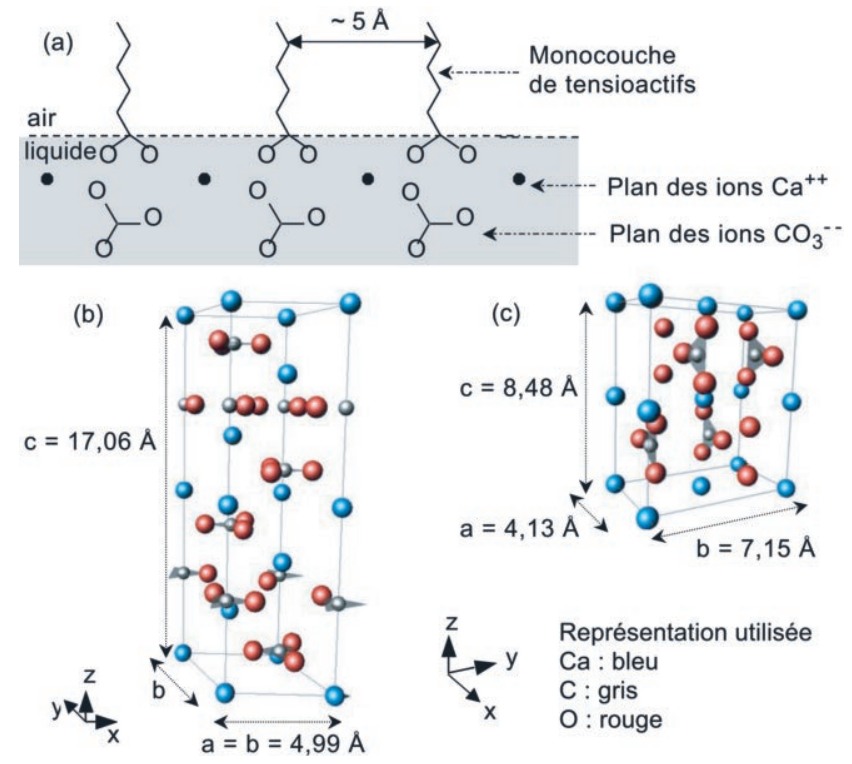

Figure 5. a) Effet stéréospécifique : la disposition des groupements carboxyliques de la monocouche favorise la nucléation de la vatérite dont les ions carbonates (plans) sont orthogonaux aux plans calcium du cristal (dessin d'après réf. [5]). b) Structure de la calcite : les plans des carbonates sont perpendiculaires à l'axe z et donc parallèles au plan $\left(\begin{array}{l}0 \\ 0\end{array}\right.$ 1). c) Structure de la vatérite : les carbonates sont parallèles à l'axe z. dente induit la formation d'un film amorphe de carbonate de calcium, rendu métastable par la présence du polymère [9].

Les questions évoquées ici dépassent bien évidemment l'étude spécifique de la nacre et de la minéralisation de $\mathrm{CaCO}_{3}$. Elles concernent l'ensemble des mécanismes de biominéralisation mis en œuvre lors de l'élaboration des matériaux biologiques composites. La réponse à de telles questions ouvrirait par ailleurs d'importantes perspectives à la fabrication de matériaux composites synthétiques, et intéressent à ce titre les ingénieurs en sciences des matériaux. Des études semblables ont ainsi été menées pour d'autres types de minéraux, sans pertinence biologique, mais précieux pour la synthèse de céramiques telles que le sulfate de baryum, le phosphate de potassium ou le sulfure de cadmium.

\section{Couplage d'organisations organique-inorganique}

Les études précédentes ignorent l'influence possible de l'état de la phase inorganique sur celui de la phase organique. Elles considèrent pour la plupart, que la structure organique n'est pas modifiée par la présence d'ions en solution et constitue une matrice figée qui détermine les conditions de minéralisation. Or, différents travaux démontrent que l'organisation de monocouches de Langmuir à l'interface air/eau peut être grandement affectée par la présence d'ions divalents en solution aqueuse (sous-phase), et ce, pour des concentrations ioniques même très faibles. Ainsi, la présence en volume de seulement 4 ions $\mathrm{Cd}^{2+}$ ou de 3 ions $\mathrm{Pb}^{2+}$ par tête polaire suffit, à $\mathrm{pH}=7,5$, à induire une contraction de la monocouche (correspondant au rapprochement et au redressement des molécules organiques) et à rigidifier fortement le film.

Ce couplage entre la structuration des phases organique et inorganique est sans doute un élément essentiel de la biominéralisation. Dans le cas de la nacre, il est possible que l'élaboration de la matrice organique dépende du niveau d'avancement de la réaction de précipitation du carbonate de calcium. Auto-assemblage de la phase organique et précipitation du carbonate de calcium suivraient ainsi des dynamiques couplées.

L'existence d'un tel couplage dynamique a été mise à profit pour réaliser la synthèse de matériaux mésoporeux présentant des pores de taille nanométrique (2 à $10 \mathrm{~nm}$ ) spatialement ordonnés. Cette synthèse fait intervenir le couplage des processus d'auto-assemblage de molécules tensio-actives et de polymérisation de précurseurs minéraux (silicates $\mathrm{Si}(\mathrm{OH})_{3} \mathrm{O}^{-}$) en solution aqueuse [10].

\section{Contrôle morphologique du minéral}

Les expériences de minéralisation au contact d'une surface organique démontrent la possibilité de contrôler le lieu de précipitation, le type cristallin et l'orientation cristallographique par le choix des molécules organiques constituant la monocouche. En revanche, dans ces expériences, la croissance du cristal dans la direction perpendiculaire au plan de la monocouche n'est limitée que par la sursaturation de la sous-phase par rapport au cristal formé. Aucun contrôle de la morphologie du cristal n'est donc envisageable, puisque définir la forme du cristal revient à limiter sa croissance dans certaines directions d'espace. Les formes minérales présentes chez les matériaux biologiques (cf. figure 2) indiquent que ce contrôle est en fait une caractéristique essentielle de la biominéralisation. Le contrôle serait exercé par des macromolécules biologiques capables de s'adsorber sélectivement sur certaines faces du cristal et d'en limiter ainsi la croissance.

Le contrôle morphologique in vivo pourrait également être lié à un processus de minéralisation en milieu confiné. Si l'on suppose que la matrice organique est formée préalablement à la précipitation du minéral, alors les espaces fermés qu'elle 
délimite pourraient permettre de définir la forme des tablettes. Aucune démonstration in vivo ne permet à ce jour de confirmer ce scénario. Pourtant, des expériences ont démontré qu'il est possible de contrôler la forme du cristal minéral grâce uniquement au confinement [11]. L'expérience consiste à induire la précipitation de carbonate de calcium à l'intérieur d'une membrane polymérique délimitant un ensemble tridimensionnel de pores de taille micrométrique. Les cristaux de carbonate de calcium adoptent alors la forme des pores de la membrane, sans relation avec les formes typiques des cristaux précipités en volume (cf. figure 6). Ces formes répondent en effet à la nécessité de minimiser l'énergie de surface en exposant les plans cristallins de plus faible énergie. Le confinement permet de retrouver la diversité des formes rencontrées chez les organismes vivants, et la forme des coques d'oursin a pu être ainsi recréée.

\section{Synthèse de matériaux bio-inspirés de type nacre}

Les travaux évoqués jusqu'à présent considèrent généralement une surface de minéralisation unique. En fait, l'élaboration d'un matériau de type nacre nécessite la présence de multiples interfaces organiques-inorganiques dont la minéralisation conduit au matériau tridimensionnel. Dans les dernières années, quelques stratégies ont été avancées pour permettre la construction en masse des structures lamellaires hybrides. Dans certains cas, les propriétés mécaniques du matériau obtenu ont pu être comparées à celles de la nacre.

- En utilisant la technique de dépôt couche à couche, une équipe de chercheurs américains a récemment produit de la « nacre » artificielle par adsorption séquentielle de tablettes d'argile, chargées négativement, et de polyélectrolytes cationiques sur substrat de silicium [12]. Le résultat est un matériau composite dont la résistance à la traction et le module d'Young se rapprochent beaucoup de ceux de la nacre naturelle ou des os lamellaires. Pour une déformation parallèle au plan des couches, la contrainte maximale de déformation de ce matériau est $\sigma=100 \pm 10 \mathrm{MPa}$, tandis que son module de Young est évalué à $\mathrm{E}=11 \pm 2 \mathrm{GPa}$. Moins d'un ordre de grandeur sépare ainsi ces valeurs de celles de la nacre $(\sigma=130 \mathrm{MPa} / \mathrm{E}=60-70 \mathrm{GPa})$ ou de l'os lamellaire $(\sigma=130 \mathrm{MPa} / \mathrm{E}=6-16 \mathrm{GPa})$.

- Une autre solution consiste à élaborer la matrice organique, puis à insérer la phase inorganique dans la matrice préformée. Dans bien des cas, cette stratégie échoue car l'insertion du minéral déstabilise la matrice et conduit à sa destruction. Elle peut cependant réussir comme le prouve la synthèse d'un composite hybride obtenu par infiltration d'une phase lamellaire de tensio-actifs par des ions cadmium, suivie d'une exposition au gaz $\mathrm{H}_{2} \mathrm{~S}$ [13].

- Les deux stratégies précédentes correspondent à des approches séquentielles : les phases organique et inorganique s'organisent successivement. Comme nous l'avons vu plus haut, il est possible de créer les conditions d'une organisation simultanée des deux phases, en couplant les dynamiques de précipitation inorganique et d'auto-organisation supramoléculaire de la phase organique. Un nanocomposite de type nacre a ainsi été formé en partant d'un mélange de molécules de silice, de tensio-actifs et de monomères organiques dans un solvant mixte eau-éthanol [14].

\section{Conclusions et perspectives}

On l'a vu, l'élaboration de matériaux par des processus biomimétiques fait appel à des mécanismes d'auto-assemblage supramoléculaire, organique et inorganique. Les connaissances proposées dans ce domaine par les physiciens et les physico-chimistes ont d'ores et déjà permis d'élaborer des systèmes modèles et de mettre en évidence différents scénarios possibles de minéralisation au contact d'une matrice organique. Pourtant, nous restons encore démunis lorsqu'il s'agit de faire la synthèse des diverses approches candidates et d'atteindre ainsi une voie de synthèse biomimétique. Au mieux, on construit de belles structures analogues du vivant, ce qui constitue déjà un succès et pourrait se révéler suffisant en termes d'application : après tout, contrairement à la peau artificielle, il n'est pas absolument nécessaire de créer une nacre en tous points semblable à l'objet naturel si seules certaines applications (tenue mécanique, ostéointégration) sont requises.

L'aspect fondamental de ces études est plus exigeant et nécessite, des physiciens, physico-chimistes et biologistes, une synthèse de leurs approches pour mieux appréhender les matériaux naturels. Cet effort de recherche est indispensable si l'on veut, à l'instar des organismes vivants, être capable de mettre au point une stratégie de construction ascendante (bottom-up) d'un matériau précis répondant à un cahier des charges. Ceci constitue l'un des défis majeurs de la science des matériaux, sous-tendu par l'intérêt technologique et industriel d'une telle maîtrise. La miniaturisation souhaitée dans tous les domaines technologiques, notamment électronique et médicale, nous demande aujourd'hui de savoir produire des matériaux structurés à l'échelle nanométrique, au-delà des limites des techniques traditionnelles de fabrication.

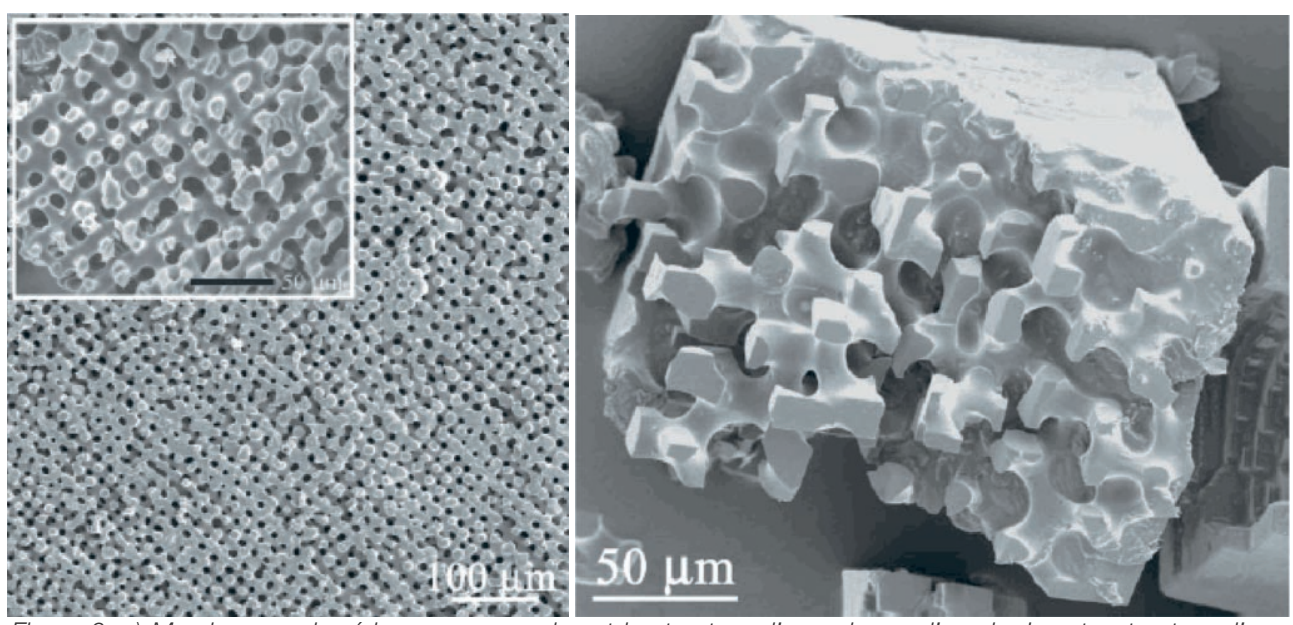

Figure 6. a) Membrane polymérique poreuse mimant la structure d'une plaque d'oursin. Insert : structure d'une plaque d'oursin. b) Le cristal formé par précipitation du $\mathrm{CaCO}_{3}$ dans cette membrane reproduit très exactement la structure de la matrice polymérique (photographies extraites de R. Park et F. Meldrum, 2004, J. Mater. Chem. 14, 2291-2296) 


\section{Références bibliographiques}

[1] Picart, C., Bulletin SFP, 155 (2006), 11-14.

[2] Giraud-Guille, M.-M., Curr. Opin. Solid State Mater. Sci., 3 (1998), 221-227.

[3] Mann, S., Biomineralization: Principles and Concepts in Bioorganic Materials Chemistry, Oxford University Press, 2001.

[4] Whitesides, G.M. et al., Proc. Natl. Acad. Sci., 99 (2002), 4769-4774.

[5] Perl-Treves, D., La Recherche, 305 (1998), 34-38.
[6] Litvin, A. et al., Adv. Mater., 9 (1997), 124-127.

[7] Mann, S. et al., Nature, 334 (1988), 692-695.

[8] DiMasi, E. et al., Cryst. Eng. Comm. 5 (2003), 346-350.

[9] DiMasi, E. et al., Langmuir, 18 (2002), 8902-8909.

[10] Monnier A. et al., Science, 261 (1993), 1299-1303.

[11] Park, R. et Meldrum, F., J. Mat. Chem., 14 (2004), 2291-2296.

[12] Tang, Z. et al., Nat. Mater., 2 (2003), 413-418.

[13] Ichinose, I. et al., J. Phys. Chem., 99 (1995), 3736-3742.

[14] Sellinger, A. et al., Nature, 394 (1998), 256-260. 